\title{
Choroidal neovascularisation induced by photocoagulation in sickle cell disease
}

\author{
P. I. CONDON, L. M. JAMPOL, * SUSAN M. FORD, \\ AND G. R. SERJEANT \\ From the Medical Research Council Laboratories, University of the West Indies, Jamaica, \\ and the * Sickle Cell Eye Clinic, Comprehensive Sickle Cell Center, University of \\ Illinois Eye and Ear Infirmary, Chicago, USA
}

SUMMARY Choroidal neovascularisation occurred in 35 out of $57(61 \%)$ patients during a trial of xenon arc photocoagulation in proliferative sickle retinopathy. Nineteen patients manifested abnormal vessel systems in the plane of the retina which tended to be benign, not enlarging, and without complications (choroidoretinal neovascularisation). In 16 patients the abnormal vessel systems grew into the vitreous, tended to increase in size, and to be associated with vitreous haemorrhage and retinal traction (choroidovitreal neovascularisation). Both forms of neovascularisation were significantly more common at younger ages. Treatment was generally unsatisfactory, frequently resulting in further enlargement of the lesions. Although the natural history of choroidal neovascularisation and its ultimate effect on visual function remains to be recorded, these lesions constitute a common and potentially serious complication of xenon arc photocoagulation in proliferative sickle retinopathy as currently performed.

The anatomy of the lesions of proliferative sickle retinopathy (PSR) with discrete feeding arteries and draining veins coursing some distance across the retina before entering the lesions renders them amenable to closure by photocoagulation. Experience with coagulation by either the xenon $\operatorname{arc}^{12}$ or the argon laser ${ }^{3}$ suggests that such therapy successfully achieves arteriolar closure in the great majority of patients. However the long-term sequelae of this therapy still requires documentation.

In a recently completed 5-year trial of the effects of xenon arc photocoagulation in proliferative sickle retinopathy ${ }^{4}$ the formation of new abnormal vessel systems occurred in the area of photocoagulation burns in 35 out of $57(61 \%)$ eyes. These vessel systems were generally unconnected to the PSR lesions destroyed by photocoagulation and were apparently supplied by choroidal vessels draining through either retinal or choroidal veins. In 19 patients these abnormal vessels were in the plane of the retina, and their fluorescence was separated from the surrounding normally perfused retina by a margin of nonfluorescence; these lesions were

Correspondence to Dr G. R. Serjeant, Medical Research Council Laboratories, University of the West Indies, Mona, Kingston 7, Jamaica, West Indies. referred to as choroidoretinal neovascularisation (CRN). In 16 patients the abnormal vessel systems projected into the vitreous, and fluorescence frequently overlay normal retina; these lesions were referred to as choroidovitreal neovascularisation (CVN).

The appearance, behaviour, complications, and response to therapy of these abnormal vessel systems during follow-up periods up to 72 months are presented in this report.

\section{Materials and methods}

Patients attended the sickle cell clinic of the University Hospital of the West Indies and participated in a trial of xenon arc photocoagulation previously described. ${ }^{24}$ Only one eye in each patient received treatment. Techniques of photocoagulation using the O'Malley Log II Photocoagulator included closure of the feeding arteriole and direct coagulation of the PSR lesion. The draining vein was not directly coagulated. Ophthalmological assessments as previously described ${ }^{5}$ were made during short-term visits (usually 3 weeks) at approximately yearly intervals by the visiting ophthalmologist (PIC). Criteria for haematological diagnosis have been summarised elsewhere. ${ }^{6}$ 
Of the original treatment group, follow-up fluorescent angiography revealed choroidal neovascularisation in $2 / 8$ patients with homozygous sickle cell (SS) disease, 27/43 patients with sickle cellhaemoglobin C (SC) disease, $4 / 4$ patients with sickle cell- $\beta^{+}$thalassaemia $\left(S \beta^{+}\right.$thal), and in $2 / 2$ patients with sickle cell- $\beta^{0}$ thalassaemia $\left(S \beta^{0}\right.$ thal). There were 22 males and 12 females with a mean age on first recognition of choroidal neovascularisation of $29 \cdot 6$ years (range 13-63 years).

\section{Results}

FACTORS RELATED TO THE DEVELOPMENT OF CHOROIDAL NEOVASCULARISATION

Time interval to diagnosis. Choroidal neovascularisation, which occurred in 35 patients, was present when next examined (9-18 months later) in 22 patients. In 1 patient subsequently found to have choroidal neovascularisation a vitreous haemorrhage did not allow accurate timing of the onset. In the remaining 12 patients initial lesions developed after a mean period of 31 months (range 16-46 months). In 1 patient $3 \mathrm{CVN}$ lesions were first diagnosed 13, 22 , and 47 months after intial therapy. The mean interval to the diagnosis of CRN lesions did not differ from that for CVN lesions. In patients with more than 1 lesion additional CRN lesions were observed to develop as late as 72 months after therapy and CVN lesions up to 50 months after therapy.

Relationship with patients' age and sex. The age of patients developing choroidal neovascularisation (mean $\pm \mathrm{SD}, 28.5 \pm 10 \cdot 6$ years) was significantly lower $(p<0.01)$ than in patients without this complication (38.7 \pm 14.6 years). Of the patients under 30 years $22 / 29(76 \%)$ developed this complication compared to $13 / 28(46 \%)$ patients above this age. There was no sex difference.

Relationship with details of therapy. The circumferential distribution of coagulation burns in patients subsequently developing choroidal neovascularisation did not differ from that in patients without this complication. The majority of choroidal lesions were in the temporal periphery, reflecting the distribution of treated PSR lesions. When a major choroidal vessel could be identified, 10/14 such vessels occurred in the mid-temporal periphery (8-10 o'clock in the right eye, 2-4 o'clock in the left eye), 2/14 in the mid-nasal periphery, and 2/14 inferiorly.

Choroidal neovascularisation did not appear to be more common in patients receiving more extensive therapy. The mean number of treatment sessions, number of arterioles treated, or number of PSR lesions treated was similar in patients develop- ing this complications and in those which did not. Neither were there significant differences in the mean number, power, or duration of photocoagulation burns between the 2 groups.

\section{OPHTHALMOSCOPIC APPEARANCE}

Lesions of choroidoretinal neovascularisation appeared as a greyish white membrane lying in the plane of the retina and circumscribed within the burn area. Abnormal vessels, when visible within the membrane, were usually straight and coarse, resembling choroidal vessels, and in some cases could be seen to arise from underlying choroidal vessels through patchy defects in the retinal pigment epithelium. Associated retinal traction or haemorrhage was unusual.

Choroidovitreal lesions differed in being whiter and more densely fibrous with less definite margins frequently overlapping the edge of the burn site. Lesions proliferated into the vitreous, causing traction on the retina in the treatment area and were frequently associated with preretinal or vitreous haemorrhages.

\section{ANGIOGRAPHIC FEATURES}

Fluorescein angiography in CRN lesions (Fig. 1a) showed a fine vessel system within the burn area, filling in the preretinal phase, and separated from the edge of the burn area by a zone of hypofluorescence presumably representing intact retinal pigment epithelium. Later phase angiograms (Figs. 1b, 2a) showed a diffuse hyperfluorescence confined to the burn area.

In CVN lesions (Figs. 2-4) early phase angiograms revealed intricate branching vessel systems projecting into the vitreous and the hyperfluorescence of later phases characteristically overlapped the edge of the treatment area. Although early filling of these vessel systems was usual, this did not always occur perhaps because of the irregular perfusion of the choroidal circulation in the equatorial region.

In 2 patients $\mathrm{CVN}$ lesions had the site and similar configuration of the initially treated PSR lesions (Fig. 5), suggesting that the choroidal vessels were now perfusing the original PSR vessel system.

BEHAVIOUR DURING FOLLLOW-UP Choroidoretinal neovascularisation initially occurred in 25 patients. No follow-up was possible in 3 patients, 1 who emigrated, 1 in whom a CRN was first observed at the last attendance, and 1 in whom the poor quality of angiograms did not allow assessment. In 6 patients $\mathrm{CRN}$ lesions developed into CVN lesions, 3 spontaneously (Figs. 2, 3) and 3 apparently after retreatment of an area of choroido- 

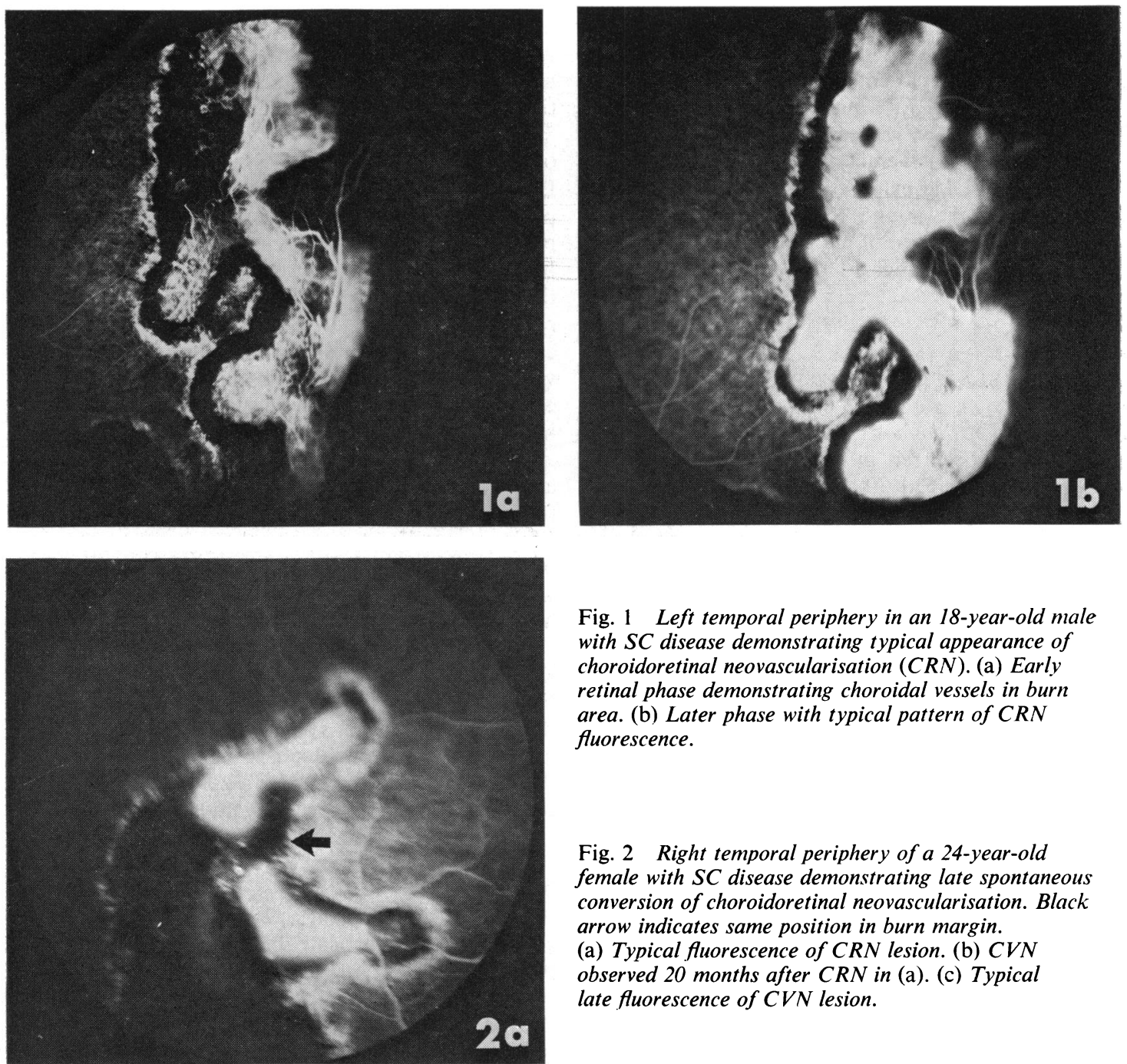

Fig. 1 Left temporal periphery in an 18-year-old male with $S C$ disease demonstrating typical appearance of choroidoretinal neovascularisation $(C R N)$. (a) Early retinal phase demonstrating choroidal vessels in burn area. (b) Later phase with typical pattern of CRN fluorescence.

Fig. 2 Right temporal periphery of a 24-year-old female with SC disease demonstrating late spontaneous conversion of choroidoretinal neovascularisation. Black arrow indicates same position in burn margin. (a) Typical fluorescence of CRN lesion. (b) CVN observed 20 months after $C R N$ in (a). (c) Typical late fluorescence of $C V N$ lesion.
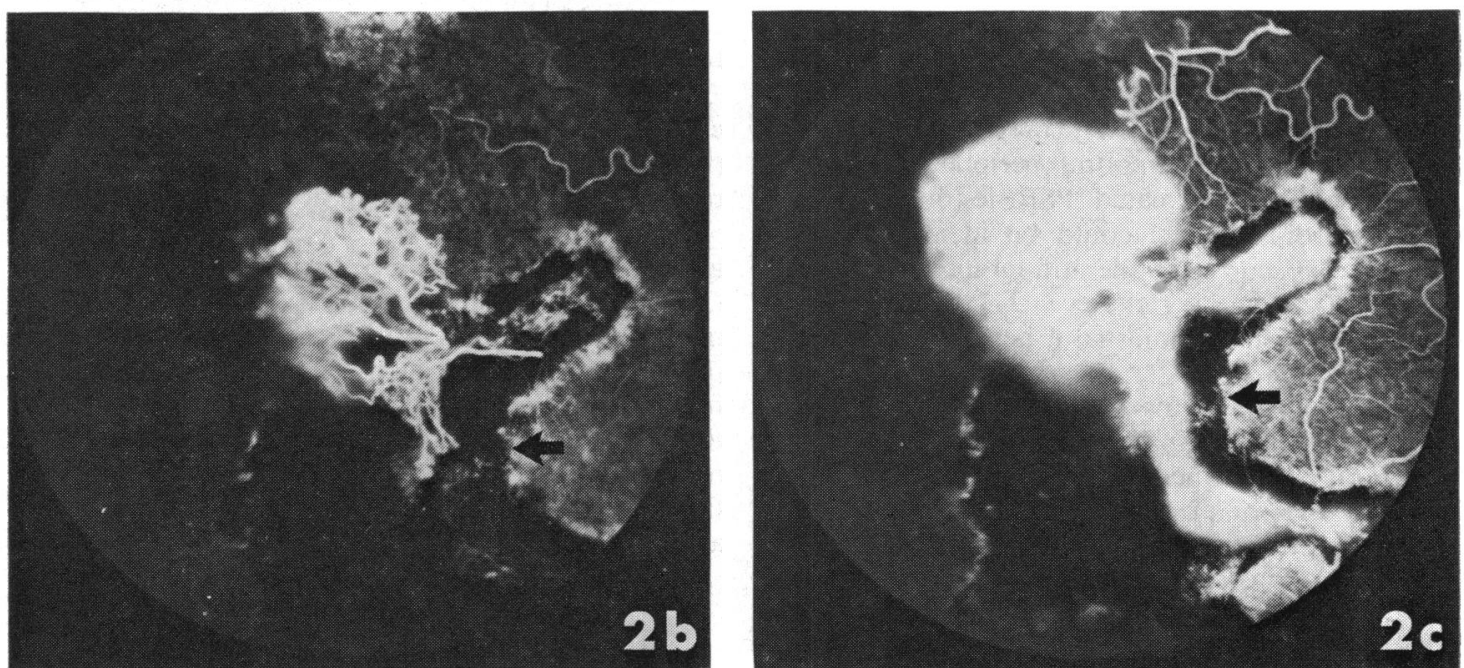



Fig. 3 Right temporal periphery in a 14-year-old male with $S C$ disease illustrating development of choroidovitreal neovascularisation from choroidoretinal neovascularisation and its apparently successful therapy by further photocoagulation. Same vessel junction indicated by straight arrow. (a) Small CRN, 12 months after photo coagulation (indicated by curved arrow). (b) CVN present 30 months after initial therapy. (c) No recurrence of CVN 17 months after retreatment.

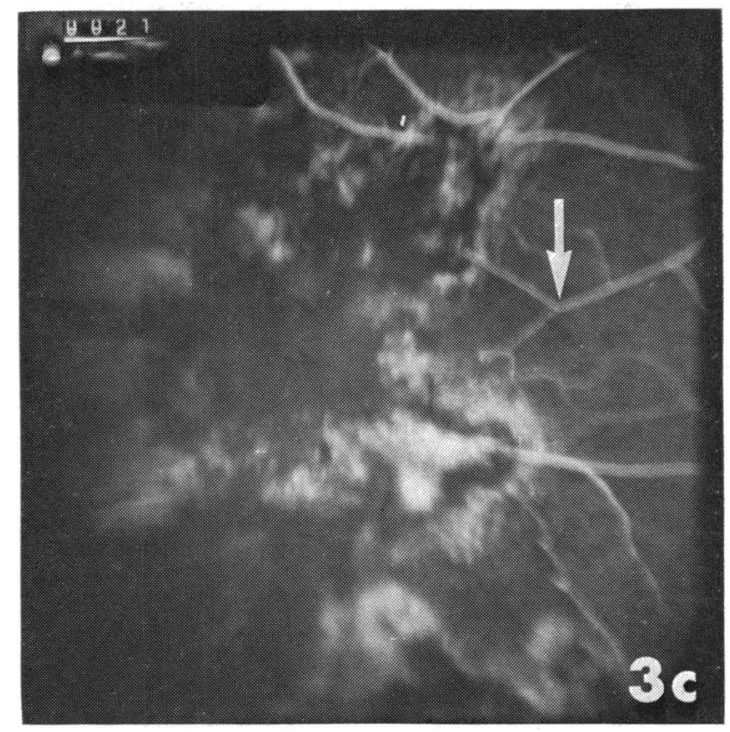

retinal neovascularisation. In the remaining 16 patients with CRNs there was a mean follow-up of 43 months (range 6-73 months). Throughout this follow-up period marked enlargement of the CRN followed treatment of the CRN lesion in 2 patients, but the remainder of CRN lesions showed no change or complications.

Of the 16 patients with choroidovitreal neovascularisation, angiograms in 1 patient manifesting 3 $\mathrm{CVN}$ lesions and in 2 others were inadequate for longitudinal assessment. In 1 patient successful therapy of the lesion (Fig. 3) forestalled longitudinal

observations. In the remaining 12 patients during a mean follow-up period of 39 months (range 6-72 months) enlargement of the CVN lesion occurred in 7 and no change in 5 . Vitreous haemorrhage occurred in 5 eyes affected by CVNs and was recurrent in 3. Increasing retinal traction occurred in 1 .

EFFECT OF THERAPY

Choroidal neovascularisation lesions were treated on 1 or more occasions in 14 patients. In 10 patients treatment of $11 \mathrm{CRN}$ lesions resulted in increasing size in 2 , conversion to $\mathrm{CVN}$ lesions in 3 , no 

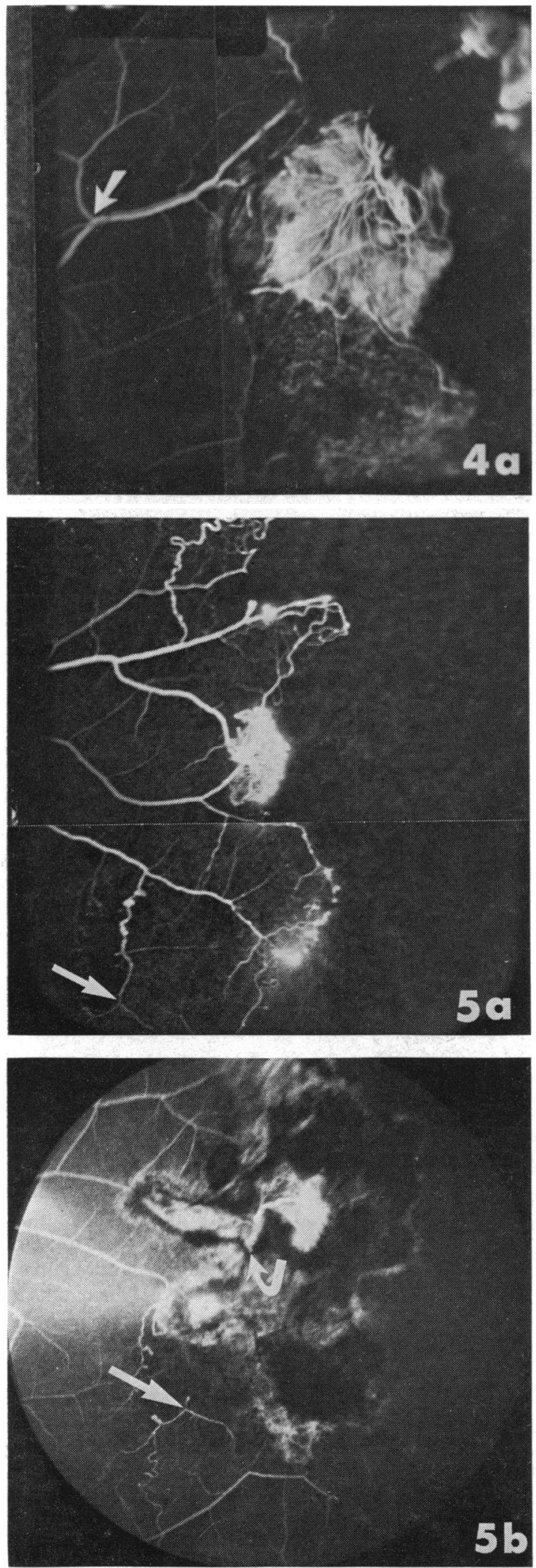

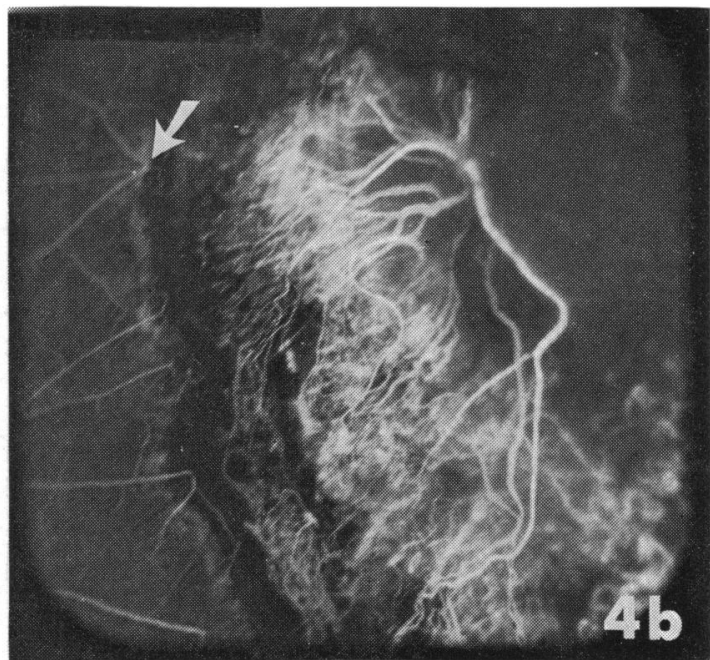

Fig. 4 Left superotemporal periphery in a 15-year-old male with $S C$ disease demonstrating rapid enlargement of choroidovitreal neovascularisation following treatment of CVN. Same vessel junction marked by arrow. (a) CVN lesion 12 months after combination of photocoagulation and diathermy to original PSR lesion. (b) Eight months after photocoagulation of CVN lesion in (a).

Fig. 5 Left temporal periphery in a 22-year-old male with $S C$ disease illustrating probable recanalisation of original PSR lesion by choroidal feeding vesselssame vessel junction indicated by straight arrow. (a) Initial PSR lesion. (b) Small CVN 18 months after therapy (choroidal feeding vessel indicated by curved arrow). NB. The CVN lesion occupies the position and has the configuration of the originally treated PSR lesion. 'c) CVN 21 months after therapy indicating rapid increase in size over 3 months.

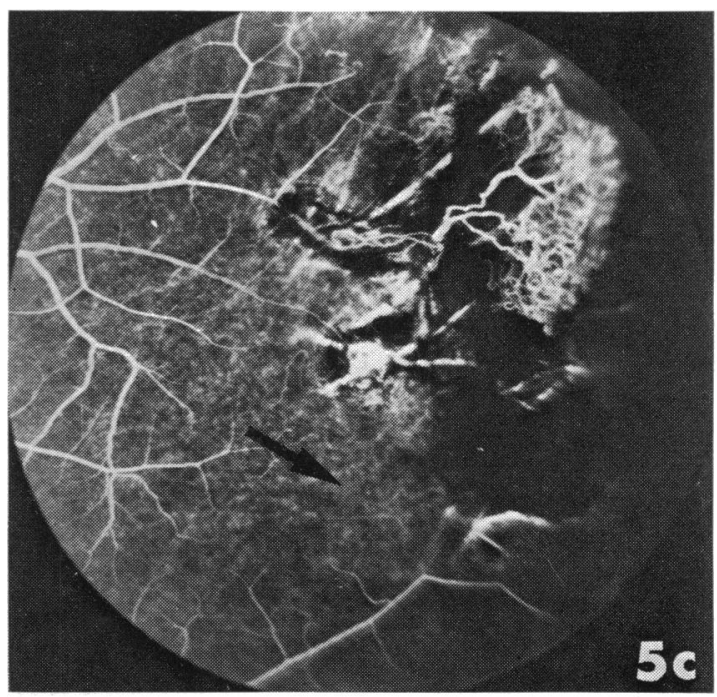


change in 2, reduction in fluorescein leakage in 2, and apparent cure of leakage in 2. However, in 2 of the 4 patients showing improvement follow-up was short, one being observed for 8 months and the other having an angioscopic assessment only 2 weeks after therapy.

Retreatment of $7 \mathrm{CVN}$ lesions in 7 patients resulted in immediate closure of feeder vessels in 3 patients. In one the lesion remained closed after 34 months follow-up but in the other 2 reperfusion was observed 2 weeks and 6 months later, and 1 of these later showed massive enlargement. Treatment of the 2 other lesions resulted in enlargement, which was massive in 1 patient. One lesion remained unchanged, and angiograms were inadequate to allow assessment in the last.

\section{Discussion}

Choroidal neovascularisation was one of the commonest complications in a recent trial of xenon arc photocoagulation in proliferative sickle retinopathy. ${ }^{4}$ It occurred in $61 \%$ of 57 patients treated between 3-7 years earlier, but the late appearance of this complication in some patients suggests that this incidence may continue to rise.

The aetiology is unclear, though it is presumed that alteration of Bruch's membrane is a prerequisite for invasion of the retina and vitreous by choroidal vessels. Such damage to Bruch's membrane may result from heat generated in the retinal pigment epithelial layer by photocoagulation and therefore might be expected to occur most frequently in patients with more intensive therapy. It was impossible to confirm such a relationship in the present study, but this may imply only inaccuracy of the indices used in estimating the amount of energy released at the retinal pigment epithelial layer. The number, power, and size of the burns gives a gross indication, but factors such as the size and location of the target area, degree of existing pigmentation, and clarity of the vitreous influence the amount of heat generated in the retina. Alternatively it could be argued that the power necessary to close arterioles in the peripheral location typical of sickle retinopathy always results in alteration of Bruch's membrane. In this case other factors would determine the invasion by choroidal vessels, and in this context it is of interest to note the significantly lower age of patients developing choroidal neovascularisation. Proliferative sickle retinopathy has been noted to progress most rapidly between the ages of 15 and 29 years, ${ }^{7}$ and it is possible that the significantly higher prevalence of choroidal neovascularisation in this age group reflects the effect of a vasoproliferative factor also responsible for proliferation of retinal vessels.

Classification of choroidal neovascularisation into choroidoretinal and choroidovitreal neovascularisations appeared to be justified by their different behaviour. Choroidoretinal lesions were generally benign, showing little tendency to progress or to give rise to complications although CRN lesions in 6 out of 22 eyes converted to CVNs. Choroidovitreal lesions on the other hand frequently increased in size and gave rise to both vitreous haemorrhage and retinal traction. Although these lesions were present when next examined (generally 1 year later) in the majority of patients, in some patients they developed as much as 6 years after the initial therapy. This late development of both CRN and CVN lesions and the spontaneous conversion of CRN to CVN lesions in 3 patients indicates that progressive vascular changes may continue remote from the time of therapy.

The results of treatment of choroidal neovascularisation were generally unsatisfactory. Although producing an apparent cure of a CVN in 1 patient, therapy tended to be followed by increasing size of both CRN and CVN lesions and treatment may actually have resulted in the conversion of CRN to CVN lesions in 3 patients.

Pathological studies of affected eyes may contribute to understanding the anatomy and pathophysiology of these abnormal vessel systems, and more information is required of the natural history of these lesions and on their effect on visual function. Until this knowledge becomes available, choroidal neovascularisation may constitute yet another reservation in photocoagulation therapy of proliferative sickle retinopathy as currently performed.

\section{References}

1 Goldberg MF. Treatment of proliferative sickle retinopathy. Trans Am Acad Ophthalmol Otolaryngol 1971; 75: 532-56.

2 Condon PI, Serjeant GR. Photocoagulation and diathermy in the treatment of proliferative sickle retinopathy. $\mathrm{Br} J$ Ophthalmol 1974; 57: 650-62.

3 Goldberg MF, Acacio I. Argon laser photocoagulation of proliferative sickle retinopathy. Arch Ophthalmol 1973; 90: $35-44$.

4 Condon PI, Serjeant GR. Photocoagulation in proliferative sickle retinopathy: results of a 5-year-old study. Br J Ophthalmol 1980; 64: 832-40.

5 Condon PI, Serjeant GR. Ocular findings in homozygous sickle cell disease in Jamaica. Am J Ophthalmol 1972; 73: 533-43.

6 Serjeant GR. The Clinical Features of Sickle Cell Disease. Amsterdam: North Holland, 1974.

7 Condon PI, Serjeant GR. Behaviour of untreated proliferative sickle retinopathy. $\mathrm{Br} J$ Ophthalmol 1980; 64: 404-11. 